\title{
Interference Rejection for Parametric Channel Estimation in Reuse-1 Cellular OFDM Systems
}

\author{
M. R. Raghavendra, Srikrishna Bhashyam, and K. Giridhar
}

\begin{abstract}
This paper proposes a joint channel-estimation method for the desired and interfering channels for cell edge users in reuse-1 orthogonal frequency-division multiplexing (OFDM) cellular systems. The following assumptions are made in proposing the algorithm: 1) The desired and interferer's channel multipath delays do not overlap; 2) the same pilot sequence is sent from the desired and interfering base stations (BSs); and 3) reuse-3 preamble symbols, as in the preamble structure in IEEE $802.16 \mathrm{~d} / \mathrm{e}$ (Wimax) systems, are used to obtain initial channel estimates without interference. If we make these assumptions, then it is possible to estimate the desired and interfering channels, even with reuse-1 pilots. The proposed pilot-based channel estimation technique exploits the delay subspace structure to reduce the impact of cochannel interference (CCI) on channel estimation. Delay subspace refers to the set of basis vectors spanning the frequency response of the desired and interfering multipath channels. This enables us to jointly estimate and track the desired and interfering channels with a lower mean-squared error (MSE), when compared with the conventional modified least-squares (mLS) technique, which ignores the structure of interference. The significance of accurate channel estimates in symbol detection schemes is demonstrated for systems employing two or more receiver antennas. The proposed channel estimator significantly improves the performance of symbol-detection schemes based on either interference nulling combiner (INC) or minimum MSE diversity combiner (MMSE-DC), when compared with detection schemes using mLS-based channel estimates. Analytical expressions are derived for the MSE of the estimated multipath delays. Simulation results are also provided to show the improved performance for the proposed channel-estimation method compared with the mLS-based channel estimation method, when used in conjunction with INC and MMSE-DC detectors.
\end{abstract}

Index Terms-Cellular orthogonal frequency-division multiplexing (OFDM), cochannel interference (CCI), estimation of signal parameters using rotational invariance method (ESPRIT), IEEE 802.16d/e, joint channel estimation, parametric channel estimation.

\section{INTRODUCTION}

$\mathbf{F}$ UTURE cellular networks based on orthogonal frequencydivision multiplexing (OFDM) are expected to be uni-

Manuscript received May 18, 2008; revised November 21, 2008 and March 11, 2009. First published March 24, 2009; current version published October 2, 2009. This work was supported by the National Research Fellowship Grant from the Institution of Electronics and Telecommunication Engineers, India, on Emerging Wireless Technologies. This paper was presented in part at the IEEE International Conference on Communications, Glasgow, U.K., June 2007. The review of this paper was coordinated by Dr. H. Artes.

M. R. Raghavendra is with Motorola India Electronics Private Ltd., Bangalore 560093, India (e-mail: raghavendramr@yahoo.com).

$\mathrm{S}$. Bhashyam and K. Giridhar are with the Telecommunication and Computer Networks Group, Department of Electrical Engineering, Indian Institute of Technology, Madras 600036, India (e-mail: srikrishna@tenet.res.in; giri@tenet.res.in).

Digital Object Identifier 10.1109/TVT.2009.2018088 versal frequency reuse (reuse-1). By cooperative scheduling of subcarriers across cells or sectors, power control, and precoding, the SIRs seen on the data subcarriers (subchannels) are likely to be higher than the corresponding SIR seen on common (broadcast) pilot subcarriers. Nearly half of the users in a reuse-1 cellular network based on the IEEE 802.16d/e wireless metropolitan access network (WMAN) standard [1] will typically see an SIR of around $0 \mathrm{~dB}$ on the broadcast pilot subcarriers [2]-[4]. Good channel estimation and tracking become very difficult for these interference-limited users.

Interference rejection techniques for data subcarriers using multiple/single-antenna systems require channel state information at the receiver. A good channel estimation and tracking method for both desired and interfering channels is required to design an efficient interference rejection combiner for multiple receive antennas. The interference suppression techniques in [8], [9], and [12] assume full channel knowledge at the receiver and do not provide the methods for channel estimation. Antenna array-based interference suppression techniques along with channel estimation have been proposed in [5]-[7] and [10]. These techniques linearly combine different antenna outputs to estimate the data, and channel estimation plays an important role in defining the combiner. While [5] and [6] use a minimum mean-squared-error (MMSE) channel estimator (which is designed for fading channel without interference, as in [25]), a pilot-based maximum-likelihood channel-estimation method capable of mitigating synchronous interference has been presented in [7]. However, this suffers from an irregular error floor in channel-estimation mean-squared error (MSE) for nonsample-spaced channels since it uses columns of discrete Fourier transform (DFT) matrix as basis functions. Another pilot-based MMSE estimation method for time-varying channels with strong cochannel interference (CCI) is presented in [10] for digital video broadcasting-terrestrial systems. This estimator exploits the pseudorandom (PR) properties of the pilot sequences on interfering transmitters in mitigating CCI. Since it models the interference components as white noise, it suffers from an irreducible error floor at high SNR. Furthermore, the performance of the channel estimator significantly degrades for nonsample-spaced channel models. A CCI suppression technique with a single receiver for coded OFDM system has been discussed in [11]. The preamble-based channel-estimation method used in [11] assumes perfect knowledge of frequency correlation (multipath delay information) of the desired channel at the receiver and assumes the channel to be time invariant over several OFDM symbols as well.

A number of parametric channel-estimation techniques for OFDM/orthogonal frequency division multiple access systems 
have been proposed for sample- and nonsample-spaced channel models [14], [16]-[18]. These parametric techniques assume a specular channel model, where each path is characterized by a delay and a complex gain. In this paper, we propose a channel estimation technique with CCI for a single- or multiplereceiver antenna system. We assume that the multipath delay locations of the desired and interferer channels do not exactly overlap. In other words, even if the power delay profiles (pdp's) are nearly the same, we assume that the time-of-flight difference between the different signals ensures that the multipath locations are distinct. From the simulations, we observe that if the delays are very close, then there is a graceful degradation in performance due to inaccurate multipath delay estimation. When the pilot sequences transmitted from the desired and interferer base stations (BSs) are same, the distinct multipath delay locations can be estimated. The proposed method estimates the multipath delays of both desired and interfering channels at the receiver. Exploiting this nonoverlapping nature of the multipath locations, it is possible to estimate and track the desired and interfering channels. ${ }^{1}$ The proposed joint channel estimator removes the error floor typically associated with DFTbased interpolation. An initial estimate of the nonoverlapping multipath locations of the desired and interfering channels is obtained using reuse- 3 preamble symbols, as in the preamble structure in IEEE 802.16d/e (Wimax) systems. However, reuse1 pilots are assumed. The interference from the same sectors (in terms of geographical orientation) of neighboring cells is neglected. Only one dominant interfering signal is considered.

This paper is organized as follows: Section II introduces the channel model and the OFDM system model. In Section III, the parametric channel-estimation algorithm is provided. Here, we discuss the steps involved in multipath delay estimation and tagging the multipath delays corresponding to the desired and interfering BSs. Section IV discusses symbol detection techniques with multiple receivers. An approximate expression for the MSE of the estimated multipath delays is derived in Section V. The MSE expression helps in deciding the minimum subarray length for satisfactory system performance. The performance of the proposed scheme is evaluated by computer simulations, and the results are provided in Section VI. Comparisons are also made with the analytical results. Section VII summarizes the main results of this paper.

\section{A. Basic Notation}

Boldface letters denote vectors or matrices; $(.)^{T},(.)^{*}$, and $(.)^{H}$ denote transpose, complex conjugate, and Hermitian, respectively; $E[$.$] denotes the expectation operator; \mathcal{C N}(\mathbf{x}, \mathbf{C})$ represent complex Gaussian vector with mean $\mathrm{x}$ and variance $\mathbf{C}$; $\mathbf{I}_{K}$ denotes the $K \times K$ identity matrix; $\mathbf{0}_{p \times q}$ denotes the

\footnotetext{
${ }^{1}$ Note that distinct multipath delays do not mean complete orthogonality between desired and interferer channels. Hence, in general, it is impossible to remove the effect of interfering channel on the desired channel estimates. However, when the channel multipath delays of both desired and interfering channels are sample spaced and "distinct," the interference can completely be removed. We provide simulation results indicating the impact of the relative path locations on the overall MSE and show that good performance is obtained, as long as one or more paths of the two channels are separated by at least $0.1 T$, where $T$ is the sampling duration of the receiver.
}

matrix of size $p \times q$ with zero entries; and $\operatorname{diag}(\mathbf{x})$ is the diagonal matrix with elements of the vector $\mathrm{x}$ on its main diagonal.

\section{OFDM SYSTEM MODEL}

Consider an OFDM system operating with a bandwidth of $B=1 / T \mathrm{~Hz}$ ( $T$ is the sampling period). The system consists of $K$ (i.e., $K$-point fast Fourier transform) subcarriers of which $K_{u}$ are useful subcarriers (excluding guard bands and dc subcarrier) with the set $\mathcal{I}_{u}$ indicating useful subcarrier positions.

The baseband equivalent of the time-varying channel impulse response is modeled as a wide-sense stationary uncorrelated scatterer zero-mean complex Gaussian process. The channel impulse response has $L$ multipath components, where each path is characterized by a complex gain factor $h_{l}$ and a delay $\tau_{l}$ [14] and has the form $h(\tau, t)=\sum_{l=0}^{L-1} h_{l}(t) \delta\left(\tau-\tau_{l}(t)\right)$, where $h_{l}(t)$ are zero-mean complex Gaussian random processes with $E\left[h_{l}(t) h_{l}^{*}(t)\right]=\sigma_{l}^{2}$ and $E\left[h_{l}(t) h_{m}^{*}(t)\right]=0$ for $l \neq m$. The paths independently fade according to the time-correlation function $E\left[h_{l}(t) h_{l}\left(t^{\prime}\right)\right]=\sigma_{l}^{2} J_{0}\left(2 \pi f_{d}\left(t-t^{\prime}\right)\right)$, where $J_{0}($.$) is$ the zeroth-order Bessel function of the first kind, and $f_{d}$ is the Doppler frequency in hertz. The channel frequency response vector for the $n$th OFDM symbol is

$$
\mathbf{H}_{n}=\mathbf{F} \mathbf{h}_{n}
$$

where $\mathbf{H}_{n}=\left[H_{0}(n), H_{1}(n), \ldots, H_{K-1}(n)\right]^{T}$, with $H_{k}(n)=$ $H\left(f=k / K T, t=n T_{s}\right)$ representing the channel frequency response on the $k$ th subcarrier of the $n$th symbol, $\mathbf{h}_{n}=$ $\left[h_{0}(n), \ldots, h_{L-1}(n)\right]^{T}$, with $h_{l}(n)=h_{l}\left(t=n T_{s}\right)$ representing the channel gain of the $l$ th path of the $n$th symbol, and $\mathbf{F}$ is the $K \times L$ sampled Fourier transform matrix with the $(k, l)$ th element given by

$$
\begin{aligned}
{[\mathbf{F}]_{k, l}=} & \exp \left(\frac{-\jmath 2 \pi k \tau_{l}}{K T}\right) \\
& \quad \text { for } k=0,1, \ldots, K-1 \quad l=0,1, \ldots, L-1 .
\end{aligned}
$$

If the variation in multipath delay locations over $N$ OFDM symbols is smaller than the resolution of the system $(1 / T)$, then we can assume the delay locations to be constant over $N$ symbols $^{2}$ (see also Section III, [17]), i.e., $\tau_{l}(t)=\tau_{l}$. Assuming sufficiently accurate frequency synchronization, the received signal vector in the frequency domain at time $n$ is

$$
\mathbf{Y}_{n}=\mathbf{X}_{n} \mathbf{H}_{n}+\mathbf{V}_{n}
$$

where $\mathbf{Y}_{n}=\left[Y_{0}(n), Y_{1}(n), \ldots, Y_{K-1}(n)\right]$ is the received vector, $\mathbf{X}_{n}$ is the diagonal matrix with data symbols $\left[X_{0}(n), X_{1}(n), \ldots, X_{K-1}(n)\right]$ on the diagonal, and $\mathbf{H}_{n}$ is the sampled frequency response of the channel during the $n$th OFDM symbol. The zero-mean complex Gaussian noise vector $\mathbf{V}_{n}$ has the distribution $\mathbf{V} \sim \mathcal{C N}\left(\mathbf{0}, \sigma^{2} \mathbf{I}_{K}\right)$. In writing (3), we have assumed the channel gains to be constant over an OFDM symbol duration, which implies that we are neglecting the

\footnotetext{
${ }^{2}$ For the system parameters considered in the simulation, we can assume $N \leq 600$.
} 


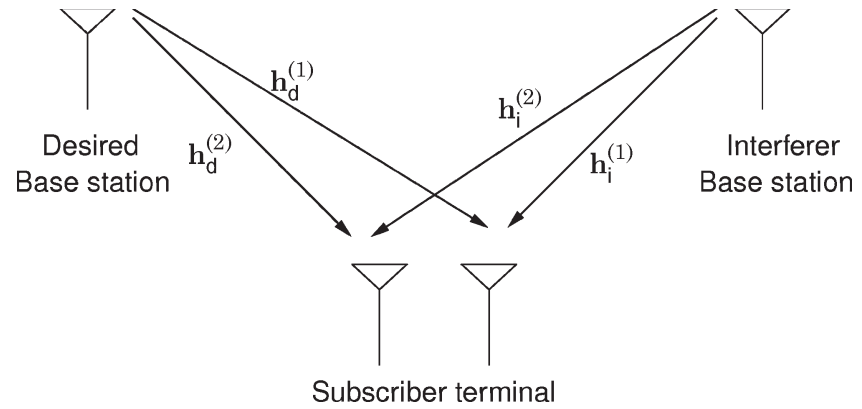

Fig. 1. OFDM system model.

intercarrier interference (ICI) due to the Doppler shift. For the normalized fade rate assumed in this paper, i.e., $f_{d} T_{s}=0.006$ (where $f_{d}$ is the fade rate in hertz, and $T_{s}$ is the OFDM symbol duration), the ICI power is upper bounded by $-42 \mathrm{~dB}$ [15]. Hence, the quasi-static assumption of the channel gains holds true for the SNRs considered in the simulation.

Consider a downlink scenario with users at cell edge or sector edge, as shown in Fig. 1. We restrict our discussion to a single interferer BS case, ${ }^{3}$ although this method can be extended for multiple interferers as well. The user receives signals from both desired and interfering BSs using $N_{r} \geq 1$ receive antennas. It is assumed that the receiver is synchronized to the desired BS, and that all the BSs are frequency and frame (time) synchronized. Let $\left\{\tau_{d}\right\}, \mathbf{h}_{d, n}^{(j)}, \mathbf{H}_{d, n}^{(j)}$ and $\left\{\tau_{i}\right\}, \mathbf{h}_{i, n}^{(j)}, \mathbf{H}_{i, n}^{(j)}$ denote the multipath delay locations (with $\tau_{d, 1}=0$ ), the channel impulse responses, and the sampled channel frequency responses of the desired and interferer channels, respectively, at the $j$ th receive antenna. Let $L=L_{d}+L_{i}$ denote the total number of multipath components. We assume that the maximum delay spread of each of the channel (with respect to $\tau_{d, 1}=0$ ) is smaller than the duration of cyclic prefix. Without loss of generality, we assume that the average signal and channel variance is unity, i.e., $E\left[|H|^{2}\right]=1$ and $E\left[|X|^{2}\right]=1$ for both desired and interfering signals. Let $K_{d}$ represent the number of subcarriers allocated to the desired user, where the set $\mathcal{I}_{d}$ indicates the corresponding subcarrier indexes. The received signal at the $j$ th receive antenna (where $j=1$ to $N_{r}$ ) in the frequency domain on the data subcarriers of the desired user at $n$th OFDM symbol is

$$
\mathbf{Y}_{n}^{(j)}=\mathbf{X}_{d, n} \mathbf{H}_{d, n}^{(j)}+\frac{1}{\sqrt{\kappa_{d}}} \mathbf{X}_{i, n} \mathbf{H}_{i, n}^{(j)}+\mathbf{V}_{n}^{(j)}
$$

where $\kappa_{d}$ represents the SIR on data subcarriers. Here, without loss of generality, we have assumed the number of receive antennas to be $N_{r}=2$. However, the channel estimation method can also be applied to multiple receivers for $N_{r}>2$. The SNR per subcarrier is defined (in decibels) as

$$
\mathrm{SNR} \triangleq \frac{E\left[\left|H_{d}\right|^{2}\right] E\left[\left|X_{d}\right|^{2}\right]}{E\left[|V|^{2}\right]}=\frac{1}{\sigma^{2}}
$$

\footnotetext{
${ }^{3}$ Since the interference in OFDM cellular systems would most often be from a single BS, we consider only one significant interfering signal in this paper. However, the presented approach is also applicable to models where there are multiple interferers
}

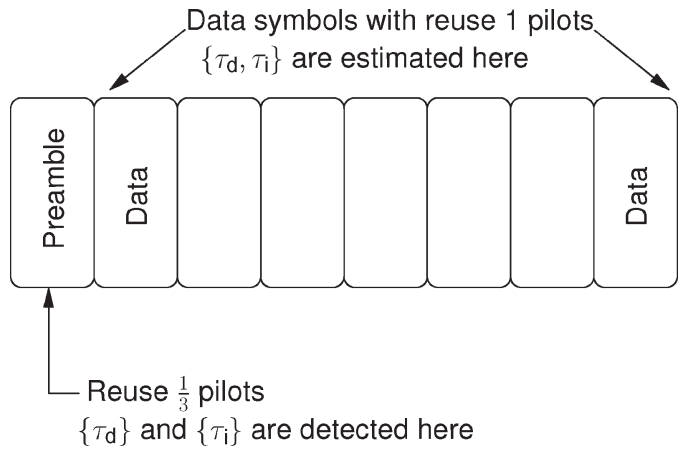

Fig. 2. OFDM frame structure.

The OFDM frame structure shown in Fig. 2 is similar to the IEEE $802.16 \mathrm{~d} / \mathrm{e}$ WMAN standard [1] and is briefly described as follows. At the start of every OFDM frame, a preamble symbol is transmitted from all BSs. Assuming BSs with a single transmit antenna ${ }^{4}$ the three BSs (one per sector) are allocated orthogonal equispaced subcarriers for the preamble (i.e., preamble symbol is based on $1 / 3$ reuse design). Let $K_{\text {pre }}$ represent the number of subcarriers allocated with the set $\mathcal{I}_{\text {pre }}$ indicating the subcarrier positions for the desired transmitter. The remaining OFDM symbols in the frame contain pilot subcarriers in reuse-1 mode that is "shared" by all BSs for channel tracking (that is, the pilot subcarrier positions fully overlap in the frequency domain). Let $K_{p}$ represent the number of pilots with the set $\mathcal{I}_{p}$ indicating their subcarrier indexes. For a fixed number of pilot subcarriers in an OFDM symbol, it has been shown that the MSE in the channel estimation is minimum if the pilots are equispaced and equipowered on the frequency grid [19], [20].

\section{Parametric Channel Estimation Using Delay Subspace Structure}

Channel "sounding" on the downlink is carried out by inserting pilot subcarriers in the time-frequency grid. In conventional channel estimation, the estimates on the pilot subcarriers are then interpolated over data subcarriers. Since the preamble symbol is reuse $1 / 3$, it is possible to estimate the channel desired and the interfering channels without any interference. The OFDM symbols following the preamble symbol also carry pilot subcarriers to enable channel tracking in fading environments. However, the pilot subcarrier positions of the desired and interferer BSs overlap, and the channel estimated at the edge of the cell can be very erroneous and cannot be used for data detection. To combat interference on pilot carriers, the use of PR pilot sequences from the desired and interferer BSs has been proposed in [1] and [29]. Due to channel distortions (introduced by multipath channel), the pseudoorthogonality between the pilot sequences is lost. While this gives some CCI mitigation, ${ }^{5}$ such schemes may not give an acceptable performance when

\footnotetext{
${ }^{4}$ The proposed method is extendable to multiple transmit antennas too, but we do not discuss it here for brevity.

${ }^{5} \mathrm{CCI}$ mitigation on channel estimates is possible, provided that the $\mathrm{CCI}$ is weak, and/or the number of pilots is much larger than the channel impulse response length.
} 
interference is strong. Henceforth, we will refer to such a channel-estimation method as the PR pilots with modified leastsquares processing (PR-mLS) technique.

In this paper, we propose to transmit the same pilot sequences from both the desired and the interferer BSs to measure the statistics of the combined channel. From the estimated statistics, the proposed method computes the multipath delays of both desired and interfering channels at the receiver. Exploiting this information, the desired and interfering channels are estimated and tracked.

The proposed channel estimation method involves the following:

1) estimating multipath delays;

2) identifying the desired and interfering multipath delays, i.e., tagging the estimated delays using preamble symbol;

3) interference rejection and channel interpolation for both desired and interfering channels.

Considering the pilot subcarrier positions on the $j$ th receive antenna, we have (the pilot vector is denoted by "overbar")

$$
\overline{\mathbf{Y}}_{n}^{(j)}=\overline{\mathbf{X}}_{d, n}^{(j)} \overline{\mathbf{H}}_{d, n}^{(j)}+\frac{1}{\sqrt{\kappa_{p}}} \overline{\mathbf{X}}_{i, n}^{(j)} \overline{\mathbf{H}}_{i, n}^{(j)}+\overline{\mathbf{V}}_{n}^{(j)}
$$

where $\kappa_{p}$ denotes the SIR on pilot subcarriers.

To estimate the channel multipath delay locations of the desired and interfering channels, the same pilot symbols are sent from both transmitters, i.e., $\overline{\mathbf{X}}_{d, n}=\overline{\mathbf{X}}_{i, n}$ with the pilot symbol energy $\left|\bar{X}_{k}(n)\right|^{2}=1$. In $802.16 \mathrm{~d} / \mathrm{e}$, pilots are unique to every cell, i.e., $\overline{\mathbf{X}}_{d, n} \neq \overline{\mathbf{X}}_{i, n}$, whereas we are proposing the use of the same pilots from both desired and interferer BSs. It will be seen later that, with the use of the same pilot sequences, it is possible to effectively mitigate $\mathrm{CCI}$ in the multipath delay domain. The least-squares (LS) channel estimates on pilot subcarriers [13] are given by

$$
\begin{aligned}
\widehat{\overline{\mathbf{H}}}_{n}^{(j)} & =\left(\overline{\mathbf{X}}_{n}^{(j)}\right)^{-1} \overline{\mathbf{Y}}_{n}^{(j)} \\
& =\overline{\mathbf{H}}_{d, n}^{(j)}+\left(1 / \sqrt{\kappa_{p}}\right) \overline{\mathbf{H}}_{i, n}^{(j)}+\left(\overline{\mathbf{X}}_{d, n}^{(j)}\right)^{-1} \overline{\mathbf{V}}_{n}^{\prime} \\
& =\overline{\mathbf{F}}_{d} \mathbf{h}_{d, n}^{(j)}+\left(1 / \sqrt{\kappa_{p}}\right) \overline{\mathbf{F}}_{i} \mathbf{h}_{i, n}^{(j)}+\underbrace{\left(\overline{\mathbf{X}}_{d, n}^{(j)}\right)^{-1} \overline{\mathbf{V}}_{n}^{\prime}}_{\overline{\mathbf{V}}_{n}^{\prime} \sim \mathcal{C N}\left(\mathbf{0}, \sigma^{2} \mathbf{I}_{K_{p}}\right)}
\end{aligned}
$$

where $\overline{\mathbf{F}}_{d}$ and $\overline{\mathbf{F}}_{i}$ are the submatrices derived by retaining rows corresponding to pilot subcarrier locations in $\mathbf{F}_{d}$ and $\mathbf{F}_{i}$, respectively. The matrices $\mathbf{F}_{d}$ and $\mathbf{F}_{i}$ are constructed as in (2) with multipath delays $\left\{\tau_{d}\right\}$ and $\left\{\tau_{i}\right\}$, respectively. Since we have assumed that the multipath delays of the desired and interferer channels do not overlap, the matrices $\mathbf{F}_{d}$ and $\mathbf{F}_{i}$ share no column in common.

The first part of the algorithm is estimating the multipath delay locations of the channels involved in (7). This equation can be viewed as the output of a sensor array with $L$ farfield narrow-band sources. The problem of multipath delay estimation in (7) is equivalent to the direction-of-arrival (DOA) estimation of different narrow-band sources. If the observed signal space exhibits the required shift invariance property, then the computationally efficient estimation of signal parameters using the rotational invariance method (ESPRIT) algorithm can be used for DOA estimation [21]. In the parametric channelestimation problem, the signal space spanned by the pilot channel estimates exhibits this shift-invariance property.

In estimating the autocorrelation matrix, we exploit the equispaced pilot structure in deriving subarrays from $\widehat{\mathbf{H}}_{n}^{(j)}$ whose correlation matrices span the same signal space [23]. Let $K_{s}$ denote the subarray size, and let $N_{s}$ denote the number of such subarrays in an OFDM symbol. The spatially smoothed autocorrelation matrix has the form

$$
\widehat{\mathbf{R}}_{s}=\frac{1}{2 N K_{s}} \sum_{j=1}^{2} \sum_{n=1}^{N} \sum_{i=1}^{K_{s}} \widehat{\mathbf{H}}_{n, i}^{(j)}\left(\widehat{\mathbf{H}}_{n, i}^{(j)}\right)^{H}
$$

where $\widehat{\hat{\mathbf{H}}}_{n, i}^{(j)}$, which is a $K_{s} \times 1$ vector, is the $i$ th subarray derived from $\widehat{\mathbf{H}}_{n}^{(j)}$. Combining forward-backward averaging with spatial smoothing, we have

$$
\widehat{\mathbf{R}}_{\mathrm{sfb}}=\frac{\widehat{\mathbf{R}}_{s}+\mathbf{J} \widehat{\mathbf{R}}_{s}^{*} \mathbf{J}}{2}
$$

where $\mathbf{J}$ is the exchange matrix (square matrix with 1 's on the anti-diagonal elements and 0's otherwise). As $N \rightarrow \infty$, the autocorrelation matrix $\widehat{\mathbf{R}}_{\mathrm{sfb}}$ converges to $1 / 2 \sum_{j=1}^{2} E\left[\widehat{\mathbf{H}}_{i}^{(j)}\left(\widehat{\mathbf{H}}_{i}^{(j)}\right)^{H}\right]=\mathbf{R}+\sigma^{2} \mathbf{I}$. The eigenvalue decomposition of $\mathbf{R}=\sum_{l=1}^{K_{s}} \lambda_{l} \mathbf{u}_{l} \mathbf{u}_{l}^{H}$, where $\lambda_{1} \geq \lambda_{2} \geq \cdots \geq \lambda_{K_{s}}$. The delay subspace of $\mathbf{R}_{\mathrm{sfb}}$ is spanned by $\mathbf{U}_{s}=\left[\mathbf{u}_{l}, \mathbf{u}_{2}, \ldots\right.$, $\left.\mathbf{u}_{L}\right]$, and the corresponding noise subspace is spanned by $\mathbf{G}_{n}=\left[\mathbf{u}_{L+1}, \ldots, \mathbf{u}_{K_{s}}\right]$.

\section{A. Estimation of Multipath Delays}

The steps involved in multipath delay estimation are described as follows:

1) Perform eigenvalue decomposition: $\widehat{\mathbf{R}}_{\mathrm{sfb}}=$ $\sum_{l=1}^{K_{s}} \widehat{\lambda}_{l} \widehat{\mathbf{u}}_{l} \widehat{\mathbf{u}}_{l}^{H}$, with $\widehat{\lambda}_{1} \geq \widehat{\lambda}_{2} \geq \cdots \geq \widehat{\lambda}_{K_{s}}$.

2) The number of paths is estimated as the number of dominant eigenvalues of $\widehat{\mathbf{R}}_{\mathrm{sfb}}$. The estimated eigenvalues are compared with the scaled noise variance $\eta \sigma^{2}$, and those eigenvalues that exceed the scaled noise variance are counted as dominant. Let $\widehat{L}$ be the number of dominant eigenvalues of $\widehat{\mathbf{R}}_{\mathrm{sfb}}$.

3) Use the eigenvectors corresponding to the $\widehat{L}$ dominant eigenvalues to form the delay subspace basis $\widehat{\mathbf{U}}_{s}=$ $\left[\widehat{\mathbf{u}}_{l}, \widehat{\mathbf{u}}_{2}, \ldots, \widehat{\mathbf{u}}_{\hat{L}}\right]$.

4) Derive the shift invariant subspaces $\widehat{\mathbf{U}}_{1}, \widehat{\mathbf{U}}_{2}$ from $\widehat{\mathbf{U}}_{s}$ as $\widehat{\mathbf{U}}_{1}=\underbrace{\left[\mathbf{I}_{K_{s}-1} \mathbf{0}_{K_{s}-1 \times 1}\right]}_{\mathbf{Z}_{1}} \widehat{\mathbf{U}}_{s}$ and $\widehat{\mathbf{U}}_{2}=\underbrace{\left[\mathbf{0}_{K_{s}-1 \times 1} \mathbf{I}_{K_{s}-1}\right]}_{\mathbf{Z}_{2}} \widehat{\mathbf{U}}_{s}$.

5) Solve for $\widehat{\Phi}$ using $\widehat{\mathbf{U}}_{2}=\widehat{\mathbf{U}}_{1} \widehat{\Phi}$.

6) Let $\widehat{\rho}_{l}$ denote the $l$ th eigenvalue of $\widehat{\Phi}$. Then, the multipath delays are estimated as

$\widehat{\tau}_{l}=\frac{\arg \left\{\rho_{l}^{*}\right\} K T}{2 \pi P}, \quad$ for $l=0,1, \ldots, \widehat{L}-1$. 
In (11), $\arg \left\{\rho_{l}^{*}\right\}$ denotes the phase angle (in the range $[0,2 \pi)$ ), and $P$ denotes the shift (in subcarriers) between the subspaces $\widehat{\mathbf{U}}_{1}$ and $\widehat{\mathbf{U}}_{2}$, which is same as the pilot spacing between the pilots in the subarrays. Note that the angle wraps around with a period of $2 \pi$. Therefore, the maximum multipath delay that can be estimated without aliasing is $K T / P$, and hence, we require $K T / P \leq \tau_{\max }$, where $\tau_{\max }=$ $\max \left\{\left\{\tau_{d}\right\},\left\{\tau_{i}\right\}\right\}$. In addition, note that the solution for $\widehat{\Phi}$ is unique only when $K_{s} \geq L+1$ ([24, pp. 1170-1171]). Given $K$ and $K_{p}$, it is possible to construct subarrays with different $P$ and $K_{s}$. Note that the pilot spacing $P$ and the subarray size $K_{s}$ fix the number of subarrays $N_{s}$. The MSE analysis of the estimated multipath delays is provided in Section V. In the subsequent section, we discuss the effect of subarray length on the MSE of the multipath delays.

The next stage of the algorithm is identifying the multipath delays corresponding to the desired and interfering channels. Since the preamble is $1 / 3$ reuse, it is possible to estimate the channel frequency response without interference on a set of carriers with indexes $\mathcal{I}_{\text {pre. }}$ The "interference free" channel estimates derived from the preamble corresponding to the desired BS are projected onto the combined basis derived from the estimated multipath delays $\left\{\widehat{\tau}_{l}\right\}_{l=0}^{\widehat{L}-1}$. The contribution along different basis vectors is then used to separate the multipath delays corresponding to the desired and interfering channels.

Let $\widehat{\mathbf{H}}_{p, d}^{(j)}$, which is a $K_{\text {pre }} \times 1$ vector, represent the "interference-free" channel estimates corresponding to the desired transmitter derived from the preamble symbol received from the $j$ th receive antenna. The interference- free channel estimates $\widehat{\mathbf{H}}_{p, d}^{(j)}$ are projected onto the combined Fourier basis derived from the estimated multipath delays as follows:

$$
\widehat{\mathbf{h}}_{p, d}^{(j)}=\left(\widetilde{\mathbf{F}}_{p}^{H} \widetilde{\mathbf{F}}_{p}\right)^{-1} \widetilde{\mathbf{F}}_{p}^{H} \widehat{\mathbf{H}}_{p, d}^{(j)}
$$

where $\widetilde{\mathbf{F}}_{p}$ is a $K_{\text {pre }} \times \widehat{L}$ matrix with $\left[\widetilde{\mathbf{F}}_{p, d}\right]_{k, l}=\exp \left(-j 2 \pi \widehat{\tau}_{l} \mathcal{I}_{\text {pre }, k}\right)$ $K T)$ for $k=1,2, \ldots, K_{\text {pre }}$ and $l=1,2, \ldots, \widehat{L}$. The vector $\widehat{\mathbf{h}}_{p, d}^{(j)}$ represents the components of $\widehat{\mathbf{H}}_{p, d}^{(j)}$ along different basis vectors associated with multipath delays. The projections $\left|\widehat{\mathbf{h}}_{p, d}^{(j)}\right|^{2}$ that exceed $\varepsilon \kappa_{p}$ are counted as dominant, where $\varepsilon$ is the user-defined parameter. Let $\mathcal{T}_{d}^{(j)}$ (which is a $\widehat{L}_{d}^{(j)} \times 1$ vector) denote the indexes of multipath delays corresponding to dominant projections. The set $\mathcal{T}_{d}^{(j)}$ represents the indexes of the desired multipath delay locations in the combined set $\left\{\widehat{\tau}_{l}\right\}_{l=0}^{\widehat{L}-1}$. Similarly, the multipath delay indexes of the interfering channel can be found.

Let $\mathcal{T}_{i}^{(j)}$ (which is a $\widehat{L}_{i}^{(j)} \times 1$ vector) represent the indexes of the multipath delay locations of the interfering channel in the combined set of multipath delays. The delay index information can be updated over time for every received preamble.

\section{B. Interference Rejection and Channel Interpolation}

Once the multipath delay locations of the desired and interfering channels are known, the delay-domain channel gains are estimated by projecting the pilot channel estimates onto the Fourier basis as follows:

$$
\widehat{\mathbf{h}}_{c, n}^{(j)}=\left(\widetilde{\overline{\mathbf{F}}}_{c}^{H} \widetilde{\overline{\mathbf{F}}}_{c}\right)^{-1} \widetilde{\overline{\mathbf{F}}}_{c}^{H} \widehat{\mathbf{H}}_{n}^{(j)}
$$

where $\quad \widetilde{\overline{\mathbf{F}}}_{c} \quad$ is $\quad$ a $\quad K_{p} \times \widehat{L} \quad$ matrix with $\left[\widetilde{\overline{\mathbf{F}}}_{c}\right]_{k, l}=$ $\exp \left(-\jmath 2 \pi \widehat{\tau}_{l} \mathcal{I}_{\mathrm{p}, k} / K T\right) \quad$ for $\quad k=1,2, \ldots, K_{p} \quad$ and $l=0,1, \ldots, \widehat{L}-1$. The desired channel impulse response estimates are the components of $\widehat{\mathbf{h}}_{c, n}^{(j)}$ with indexes $\mathcal{T}_{d}^{(j)}$, i.e., $\widehat{\mathbf{h}}_{d, n}^{(j)}=\widehat{\mathbf{h}}_{c, n}^{(j)}\left(\mathcal{T}_{d}^{(j)}\right)$. The interfering channel impulse response estimates are the components of $\widehat{\mathbf{h}}_{c, n}^{(j)}$ with indexes $\mathcal{T}_{i}^{(j)}$, or $\widehat{\mathbf{h}}_{i, n}^{(j)}=\widehat{\mathbf{h}}_{c, n}^{(j)}\left(\mathcal{T}_{i}^{(j)}\right)$. Finally, we derive frequency-domain channel estimates on the subcarriers of the desired and interfering BS using

$$
\begin{aligned}
\widehat{\mathbf{H}}_{d, n}^{(j)} & =\widetilde{\mathbf{F}}_{d} \widehat{\mathbf{h}}_{d, n}^{(j)} \\
\widehat{\mathbf{H}}_{i, n}^{(j)} & =\widetilde{\mathbf{F}}_{i} \widehat{\mathbf{h}}_{i, n}^{(j)}
\end{aligned}
$$

where $\quad \widetilde{\mathbf{F}}_{d}$ is a $K_{d} \times \widehat{L}_{d}^{(j)}$ matrix with $\left[\widetilde{\mathbf{F}}_{d}\right]_{k, l}=$ $\exp \left(-\jmath 2 \pi \widehat{\tau}_{l} \mathcal{I}_{\mathrm{d}, k} / K T\right)$ for $k=1,2, \ldots, K_{d}$ and $l \in \mathcal{T}_{d}^{(j)}$, and $\widetilde{\mathbf{F}}_{i}$ is a $K_{d} \times \widehat{L}_{i}$ matrix with $\left[\widetilde{\mathbf{F}}_{i}\right]_{k, l}=\exp \left(-\jmath 2 \pi \widehat{\tau}_{l} \mathcal{I}_{\mathrm{i}, k} / K T\right)$ for $k=1,2, \ldots, K_{d}$ and $l \in \mathcal{T}_{i}$. The channel estimates $\widehat{\mathbf{H}}_{d, n}^{(j)}, \widehat{\mathbf{H}}_{i, n}^{(j)}$ can further be improved by exploiting the time correlation of the temporal channel estimates $\widehat{\mathbf{h}}_{d, n}^{(j)}$ and $\widehat{\mathbf{h}}_{i, n}^{(j)}$, respectively, of the finite-impulse-response (FIR) filter. The coefficients of the FIR filter are derived using Weiner filter theory [25].

\section{Symbol Detection With Multiple Receivers}

To show the importance of the proposed channel-estimation method, we consider the effect of channel estimation on two different symbol detection techniques that are possible with diversity-spaced receive antennas. The data symbols are detected by linearly combining the outputs of multiple receive antennas. The accuracy of channel estimation plays an important role in defining an efficient linear combiner. It is clear from the simulations provided in Section VI for high-interference scenarios that the proposed channel-estimation method clearly outperforms the mLS-based method at all SNRs.

\section{A. Minimum Mean Square Error Diversity Combiner}

The diversity combiner (DC) reduces the MSE between the transmitted and estimated symbols. The MMSE-DC requires and takes the form of [6], [25]

$$
\widehat{X}_{\mathrm{d}, k}(n)=\mathbf{w}_{k}^{H}(n) \mathcal{Y}_{k, n}
$$

where $\mathcal{Y}_{k, n}=\left[\begin{array}{ll}Y_{k}^{(1)}(n) & Y_{k}^{(2)}(n)\end{array}\right]^{T}$, and the weight vector $\mathbf{w}_{n}(k)$ is derived as

$$
\mathbf{w}_{n}(k)=\mathbf{R}_{n}(k)^{-1} \mathbf{H}_{d, n}(k)
$$


with $\mathbf{R}_{n}(k)=E_{c}\left[\mathcal{Y}_{k, n}\left(\mathcal{Y}_{k, n}\right)^{H}\right]$, where $E_{c}$ represents the conditional expectation given the channel parameters corresponding to both desired and interferer. Observe that MMSE-DC needs the desired channel and interferer channel information. It is to be noted that in the absence of interference, the maximal ratio combining and the MMSE-DC are equivalent.

\section{B. Interference Nulling Combiner}

To combat the interference with multiple receivers, an interference-nulling (zero-forcing) combining solution can be derived. The set of equations on the $k$ th subcarrier in (4) for $j=1,2$ can be written as

$$
\mathcal{Y}_{k, n}=\mathcal{H}_{k, n} \mathcal{X}_{k, n}+\mathcal{V}_{k, n}
$$

where

$$
\begin{aligned}
\mathcal{H}_{k, n} & =\left[\begin{array}{ll}
H_{\mathrm{d}^{(1)}, k}(n) & H_{\mathrm{i}^{(1)}, k}(n) \\
H_{\mathrm{d}^{(2)}, k}(n) & H_{\mathrm{i}^{(2)}, k}(n)
\end{array}\right] \\
\mathcal{X}_{k, n} & =\left[\begin{array}{ll}
X_{\mathrm{d}, k}(n) & X_{\mathrm{i}, k(n)}
\end{array}\right] \\
\mathcal{V}_{k, n} & =\left[\begin{array}{ll}
V_{k}^{1}(n) & V_{k}^{(2)}(n)
\end{array}\right]^{T} .
\end{aligned}
$$

An equalizer is defined to null the effect of interference at the receiver, i.e., we design an equalizer $\mathcal{G}_{k, n}$ such that

$$
\mathcal{G}_{k, n}^{H} \mathcal{H}_{k, n}=\left[\begin{array}{ll}
1 & 0
\end{array}\right]
$$

and an estimate of the desired symbol is given by

$$
\widehat{X}_{\mathrm{d}, k}(n)=\mathcal{G}_{k, n}^{H} \mathcal{Y}_{k, n} .
$$

Observe that similar to MMSE-DC, the interference nulling combiner (INC) also requires the channel state information of both desired and interferer channels.

In the next section, we derive analytical expressions for the MSE of the multipath delay estimates.

\section{MSE Analysis of Multipath Delays}

Here, we derive the expressions for the MSE of multipath delays estimated from the ESPRIT algorithm. As mentioned earlier, the problem of multipath delay estimation is equivalent to DOA estimation in array processing. The statistical performance of the ESPRIT-based DOA estimator is discussed in [34]. The MSE analysis provided in [34] exploits no autocorrelation smoothing in estimating the DOA. It is observed that with a uniform linear array, exploiting smoothing methods such as spatial smoothing and forward-backward averaging in estimating the autocorrelation matrix gives a significant improvement in the convergence performance of the DOA estimators [22].

Multipath delay estimation using ESPRIT involves finding eigenvalues $\{\lambda\}$ of the $\Phi$ matrix, as explained in Section III-A. Due to the finite averaging effects, the autocorrelation matrix $\mathbf{R}$ and, hence, the multipath delays $\{\tau\}$ are estimated in error. A closed-form MSE expression for the multipath delays estimated from the erroneous autocorrelation matrix is derived in [32] and [34]. The analysis is carried out by using the first-order perturbation theory of subspaces. The MSE expression derived here follows the procedure given in [32] and [34].

From [33] and [34], the error in the estimation of the $l$ th eigenvalue in (11) $\Delta \rho_{l}=\rho_{l}-\widehat{\rho}_{l}$ is expressed as a function of errors in delay subspace $\Delta \mathbf{U}_{s}$ as

$$
\Delta \rho_{l}=\mu_{l}^{H} \mathbf{U}_{1}^{+}\left(\mathbf{Z}_{2}-\rho_{l} \mathbf{Z}_{1}\right) \mathbf{G G}^{H} \Delta \mathbf{U} \nu_{l}
$$

where $\mu_{l}^{H}$ and $\nu_{l}$ are the left and right eigenvectors of $\Phi$ corresponding to the eigenvalue $\rho_{l}$, such that $\mu_{l}^{H} \nu_{l}=1$, and $\Delta \mathbf{U}=\mathbf{U}_{s}-\widehat{\mathbf{U}}_{s}$. The first-order perturbation of the errors in the estimated signal eigenvectors projected onto the noise subspace space is given by [32]

$$
\mathbf{G G}^{H} \Delta \mathbf{U}_{s}=\mathbf{G G}^{H} \widehat{\mathbf{R}}_{\mathrm{sfb}} \mathbf{R}^{+} \mathbf{U}_{s} .
$$

Substituting (22) in (21), we can write the error in the $l$ th eigenvalue as

$$
\Delta \rho_{l}=\zeta_{l}^{H} \mathbf{G G}^{H} \widehat{\mathbf{R}}_{\mathrm{sfb}} \eta_{l}
$$

where $\zeta_{l}^{H}=\mu_{l}^{H} \mathbf{U}_{1}^{+}\left(\mathbf{Z}_{2}-\rho_{l} \mathbf{Z}_{1}\right)$, and $\eta_{l}=\mathbf{R}^{+} \mathbf{U}_{s} \nu_{l}$. It is clear that $\eta$ is in the signal subspace $\mathbf{U}_{s}$. It has been shown in [34] that the vector $\zeta_{l}$ is in the noise subspace $\mathbf{G}$, which implies that $\zeta_{l}^{H} \mathbf{G G}^{H}=\zeta_{l}^{H}$.

The MSE in the estimate of the $l$ th eigenvalue $\rho_{l}$ is given by

$$
E\left[\left|\Delta \rho_{l}\right|^{2}\right]=E\left[\left(\zeta_{l}^{H} \widehat{\mathbf{R}}_{\mathrm{sfb}} \eta_{l}\right)\left(\zeta_{l}^{H} \widehat{\mathbf{R}}_{\mathrm{sfb}} \eta_{l}\right)^{*}\right]=\Gamma_{\zeta_{l} \zeta_{l} \eta_{l} \eta_{l}}
$$

where

$$
\Gamma_{\alpha \beta \gamma \delta}=\frac{\gamma^{H} \Theta_{\alpha \beta} \delta}{4 N N_{s}^{2}}
$$

and $\Theta_{\alpha \beta}=\Theta_{1}+\Theta_{2}+\Theta_{3}+\Theta_{4}$, where $\alpha, \beta, \gamma$, and $\delta$ are $K_{s} \times 1$ vectors, i.e.,

$$
\begin{aligned}
& \Theta_{1}=\sum_{p=1}^{N_{s}} \sum_{q=1}^{N_{s}}\left(\alpha^{H} \mathbf{R}_{p q} \beta\right) \mathbf{R}_{q p} \\
& \Theta_{2}=\sum_{p=1}^{N_{s}} \sum_{q=1}^{N_{s}} \mathbf{J R}_{p q}^{T} \alpha^{*} \beta^{T} \mathbf{J R}_{q p} \\
& \Theta_{3}=\sum_{p=1}^{N_{s}} \sum_{q=1}^{N_{s}}\left(\alpha^{H} \mathbf{J R}_{q p}^{T} \mathbf{J} \beta\right) \mathbf{J} \mathbf{R}_{p q}^{T} \mathbf{J} \\
& \Theta_{4}=\sum_{p=1}^{N_{s}} \sum_{q=1}^{N_{s}} \mathbf{R}_{q p} \mathbf{J} \alpha^{*} \beta^{T} \mathbf{R}_{p q}^{T} \mathbf{J}
\end{aligned}
$$

and $\mathbf{R}_{p q}$ is the $K_{s} \times K_{s}$ correlation matrix between the $p$ th and $q$ th subarray, i.e., $\mathbf{R}_{p q}=1 / 2 \sum_{j=1}^{2} E\left[\widehat{\widehat{\mathbf{H}}}_{p, n}^{(j)}\left(\widehat{\mathbf{H}}_{q, n}^{(j)}\right)^{H}\right]$. The MSE in the $l$ th multipath delay $\Delta \tau_{l}$ and the MSE in the $l$ th eigenvalue $\Delta \rho_{l}$ are related by [33]

$$
E\left[\left(\Delta \tau_{l}\right)^{2}\right]=\left(\frac{K}{2 \pi P}\right)^{2}\left(\Gamma_{\zeta \zeta \eta \eta}-\Re\left\{\Gamma_{\zeta \eta \zeta \eta}\right\}\right) .
$$


The total MSE in the multipath delay estimate is defined as

$$
\operatorname{MSE}(\tau)=L \sum_{l=1}^{L} \sigma_{l}^{2} E\left[\left(\Delta \tau_{l}\right)^{2}\right]
$$

where $\sigma_{l}^{2}$ is the variance of the $l$ th path with $\sum_{l=1}^{L} \sigma_{l}^{2}=1$. Given the system parameters, $\operatorname{MSE}(\tau)$ can analytically be evaluated by using (28).

\section{A. Discussion on the Choice of Subarray Size}

In Section III, we have seen that dividing the pilot channel estimates into a number of subarrays increases the autocorrelation averaging, which helps in multipath delay estimation. In this section, we discuss the effect of subarray size on the MSE of the multipath delay estimate. The MSE of the multipath delay estimate is a function of the subarray length. It is clear from array processing theory that, to estimate the multipath delays of a channel with $L$ paths, the minimum length of subarray required is $L+1$ ([24, pp. 1170-1171]). To improve the autocorrelation estimation, it seems obvious to choose subarrays of minimum lengths, since it offers greater autocorrelation averaging. However, the minimum subarray sizes may not always result in minimum $\operatorname{MSE}(\tau)$, as $\operatorname{MSE}(\tau)$ also depends on the eigenvalue spread of the autocorrelation matrix $\mathbf{R}$, as reflected in (22). The expression for perturbation in the signal subspace as in (22) involves the pseudoinverse of the $\mathbf{R}$ matrix, and hence, the perturbation will be more when the eigenspread of the matrix $\mathbf{R}$ is large. The eigenvalue spread of the autocorrelation matrix is a function of frequency selectivity of the channel over the length of subarray and, hence, is a function of the channel delay spread. For smaller subarray sizes $\left(K_{s} \geq L+1\right)$, the eigenvalue spread is very large and results in a larger $\operatorname{MSE}(\tau)$. However, with the increase in subarray length, the eigenvalue spread decreases, and hence, $\operatorname{MSE}(\tau)$ decreases. The simulation and analytical results related to the MSE of the multipath delay estimator $\operatorname{MSE}(\tau)$ are provided next.

\section{RESUlTS AND DisCUSSION}

The performance of the proposed estimator is also evaluated using computer simulations. An OFDM system is simulated with the following parameters [1]: center frequency $f_{c}=2.2 \mathrm{GHz}$, bandwidth $B=1 / T=5 \mathrm{MHz}$ (where $T$ is the sampling time), total number of subcarriers used $K=512$, number of useful subcarriers $K_{u}=420$, duration of the cyclic prefix $L_{\mathrm{cp}} T=12.8 \mu \mathrm{s}$ (64 samples), and $T_{s}=\left(K+L_{\mathrm{cp}}\right) T$ is the OFDM symbol duration. Each transmitter is allocated $K_{\text {pre }}=420 / 3=140$ nonoverlapping equispaced subcarriers over the preamble symbol, and the subsequent data symbols are allocated $K_{p}=53$ pilots (with reuse-1). The desired user is allocated 50 data subcarriers over the downlink. The userdefined parameters are set at $\eta=1.2$ and $\varepsilon=0.05$. The subarray size and the number of subarrays are given by $K_{s}=30$ and $N_{s}=23$, with the pilot spacing in the subarray being $P=8$. Each OFDM frame consists of 50 OFDM symbols. A preamble symbol is transmitted at the start of every frame and is followed

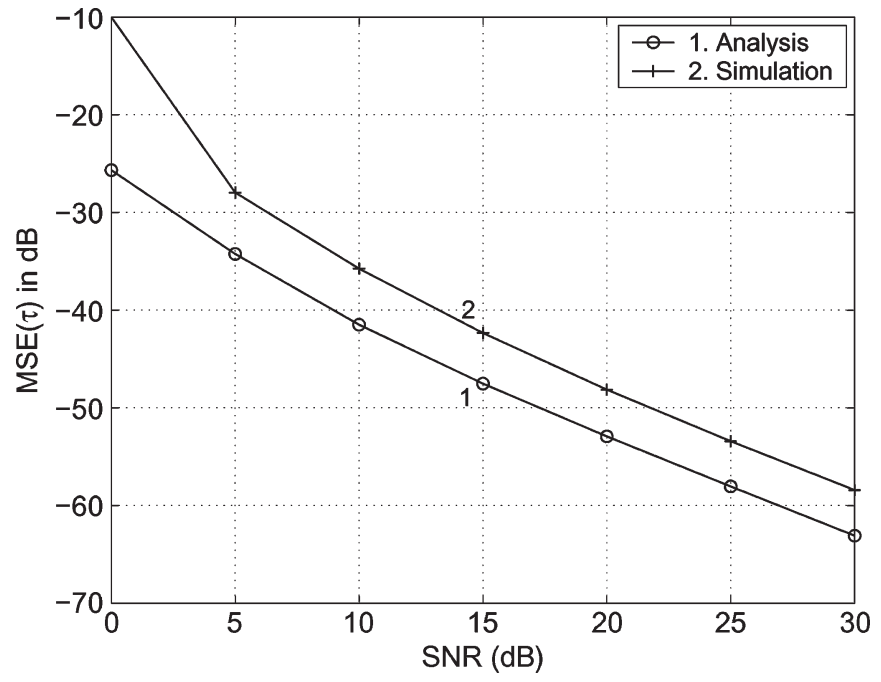

Fig. 3. Plot comparing the simulated and analyzed $\operatorname{MSE}(\tau)$ for autocorrelation averaging over 50 OFDM symbols.

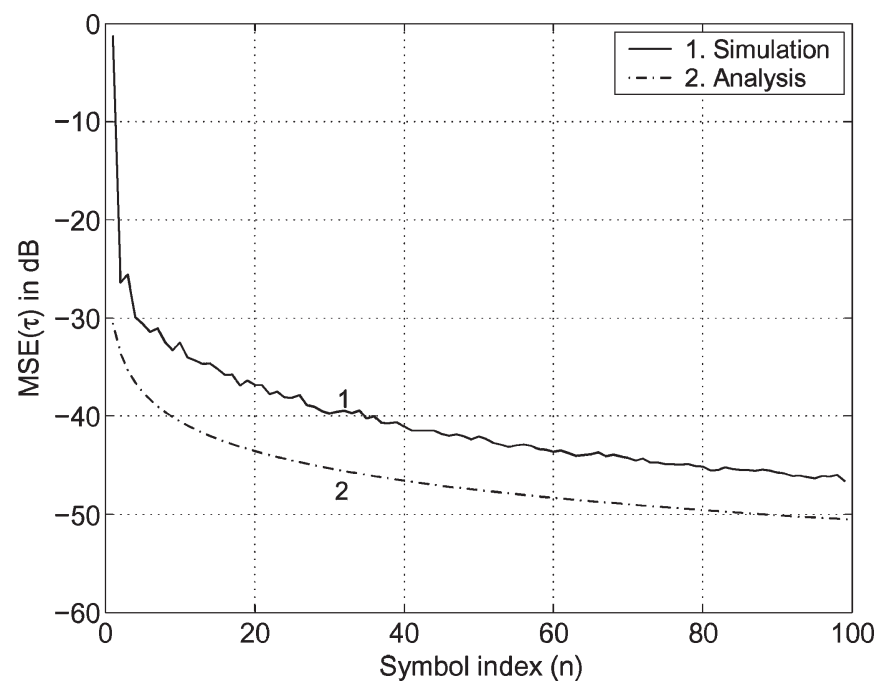

Fig. 4. $\operatorname{MSE}(\tau)$ convergence plot with different autocorrelation averaging.

by data symbols. In all our simulations, the channel pdp is assumed to be exponential with $\operatorname{pdp}(\tau) \propto \exp (-0.1 \tau)$.

We have considered a four-tap channel with Rayleigh coefficients for the desired and interferer signals with multipath delays uniformly distributed over $\left[\begin{array}{ll}0 & 7.2 \mu \mathrm{s}\end{array}\right]$ (exponential pdp).

The maximum delay difference between the desired signal and the interferer signal is within the duration of the cyclic prefix. The nonoverlapping multipath delays are randomly generated. For the simulation results provided in Figs. 3-9, the multipath delays are randomly generated with a minimum spacing of $0.25 T$ between any two paths. However, we have also presented the MSE and bit error rate (BER) results when the minimum spacing between randomly generated delays is progressively reduced to zero.

The pdp of the channel is assumed to be exponentially decaying. The channel paths independently fade according to Jakes' power spectrum [28]. The normalized fade rate is $f_{d} T_{s}=0.006$ (vehicular speed $=10 \mathrm{~m} / \mathrm{s}$ ). A four-tap FIR filter designed with exact knowledge of the Doppler power 


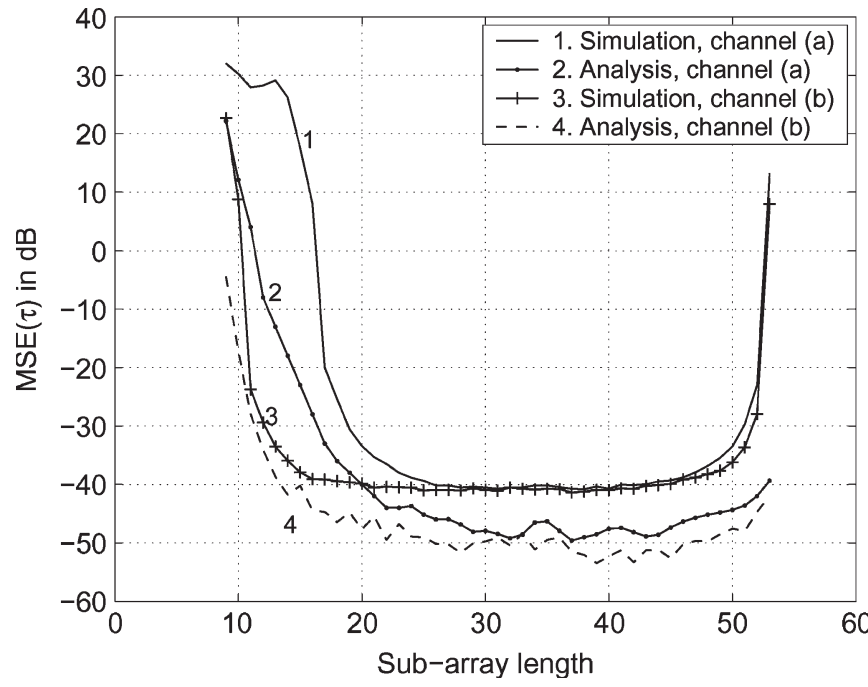

Fig. 5. $\operatorname{MSE}(\tau)$ as a function of subarray length $K_{s}$.

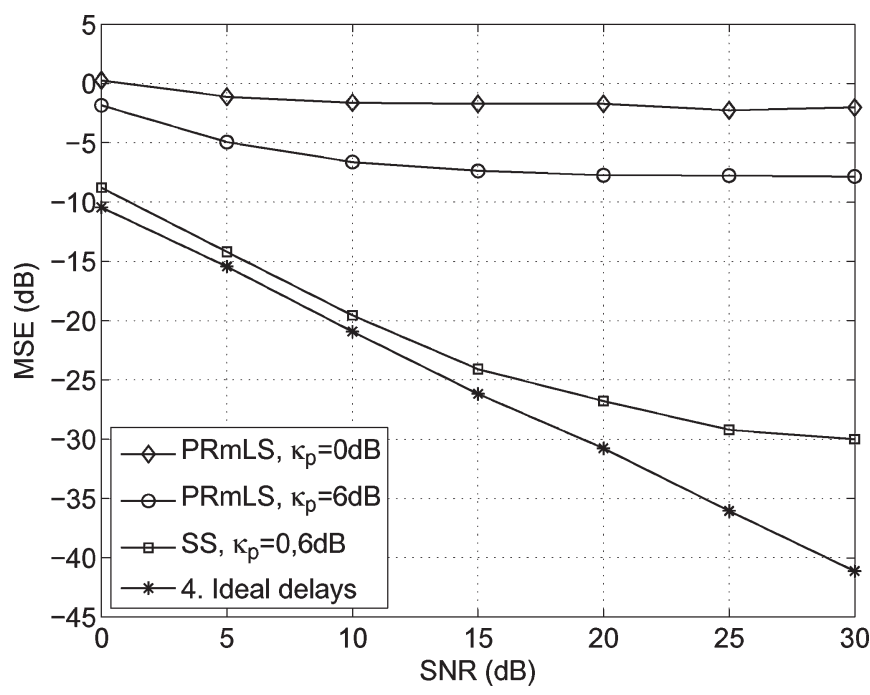

Fig. 6. MSE convergence plot comparing the proposed SS method with the PR-mLS-based method.

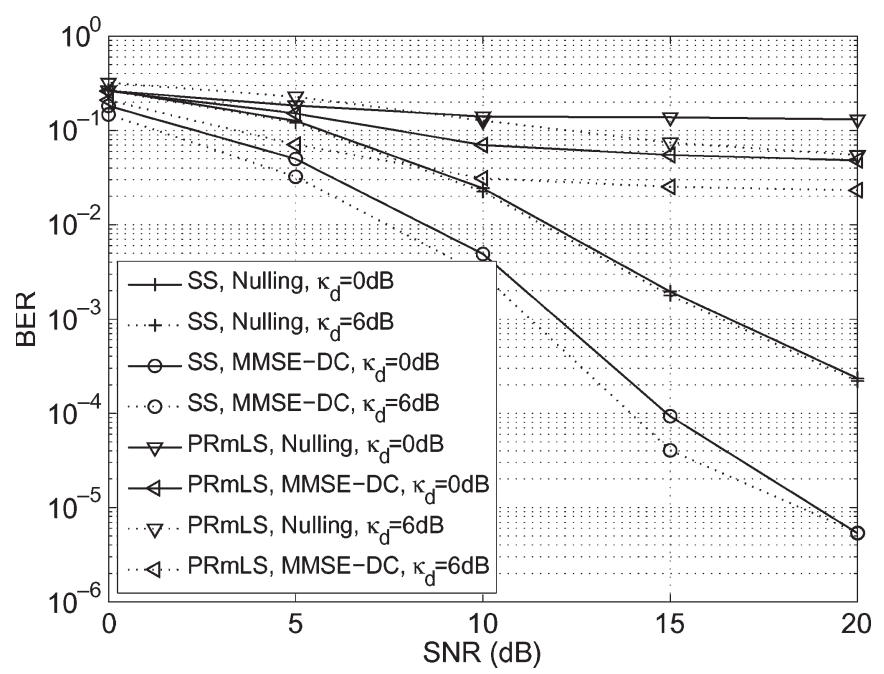

Fig. 7. Coded BER performance comparison of the different channel estimation techniques for $\kappa_{p}=0 \mathrm{~dB}$.

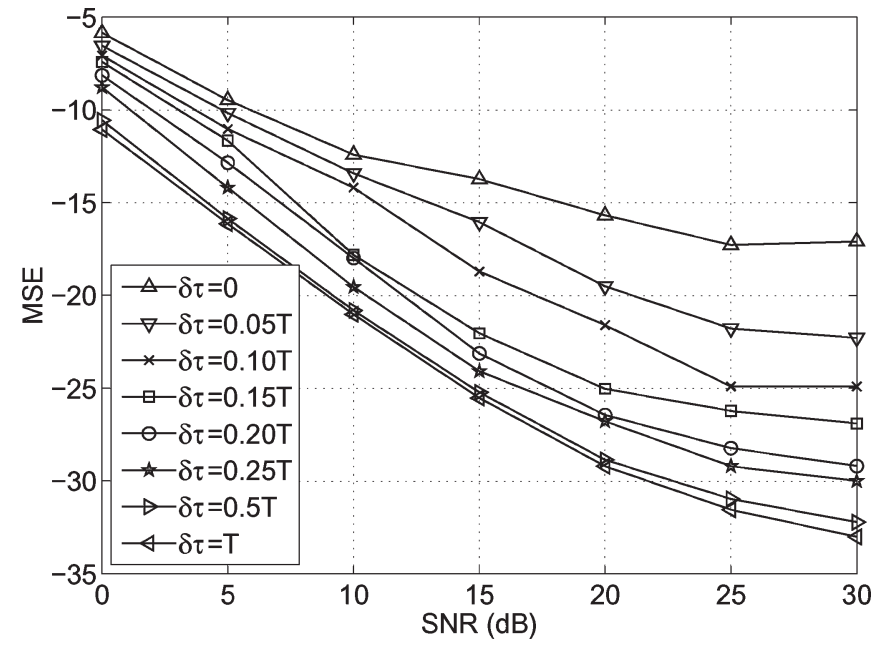

Fig. 8. MSE convergence plot for the proposed SS method for different multipath delay spacing $\delta \tau$.

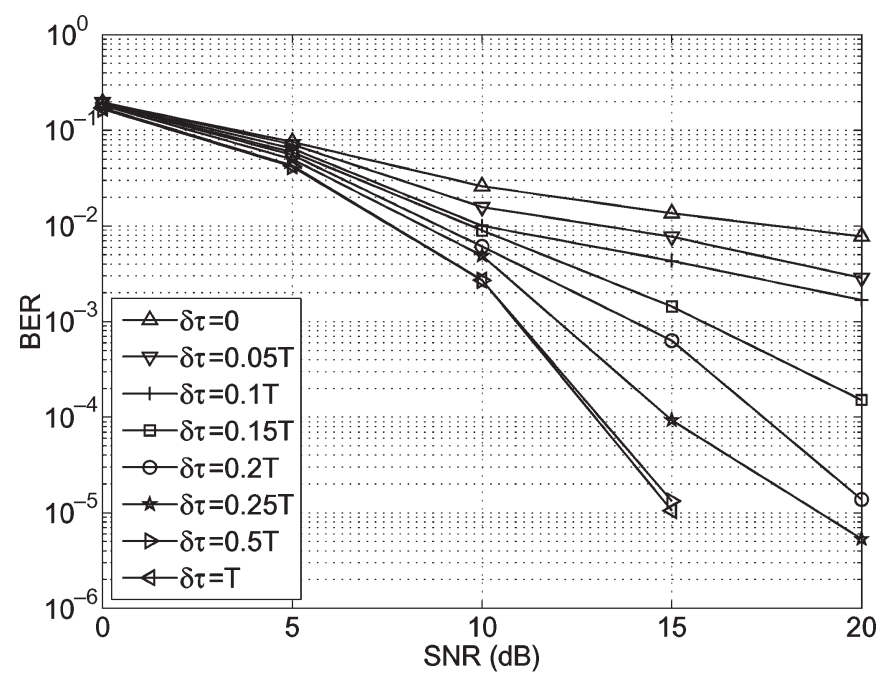

Fig. 9. Coded BER performance comparison for different multipath delay spacing $\delta \tau$.

spectrum is used to exploit the time correlation in the channel (it is found that increasing the filter length greater than 4 gives a rather negligible improvement in MSE performance [31, pp. 535-539]).

The performance of the algorithm is evaluated using MSE and BER by averaging over channels with different multipath delay locations. For the coded OFDM system, the input bits are encoded with rate $1 / 2$ parallel concatenated convolutional turbo codes, as explained in [1, Sec. 8.4.9] and then modulated using the QPSK scheme. The synchronous interferer signal is also assumed to be QPSK modulated. The channel MSE is defined as (for some $n$ )

$$
\mathrm{MSE}=\frac{1}{2 K_{d}} \sum_{j=1}^{2} \sum_{k \in \mathcal{I}_{d}} E\left\{\left|H_{d, n}^{(j)}(k)-\widehat{H}_{d, n}^{(j)}(k)\right|^{2}\right\} .
$$

Fig. 3 shows the MSE performance of the multipath delay estimator for $N=50$ symbols. The analytical MSE, as derived from (28), considers first-order perturbation analysis and independent channel realizations. However, in the simulations, 
the multipath delays are estimated from the correlated channel with a normalized fade rate of $f_{d} T_{s}=0.006$. We observe here that the simulated MSE is around $5 \mathrm{~dB}$ away from the analytical MSE. Fig. 4 compares the simulated and analytical MSE convergence performance for different $N$ with the SNR fixed at $15 \mathrm{~dB}$. For a satisfactory system operation, we can fix $\operatorname{MSE}(\tau)$ and then decide the number of symbols required for averaging.

In Fig. 5, the effect of the subarray size as the multipath delay MSE is presented. The simulation and analysis are carried out at $15-\mathrm{dB}$ SNR. The MSE difference between the simulation and the analysis is due to the fact that the analysis uses firstorder perturbation theory and independent channel realizations in deriving a closed-form expression for $\operatorname{MSE}(\tau)$. The subarray length is varied from $L+1=9$ to $K_{p}=53$. The $\operatorname{MSE}(\tau)$ initially reduces with the increase in subarray length and then floors at approximately $-40 \mathrm{~dB}$ over a range of subarray lengths (between 25 and 45 in the plot). To demonstrate the effect of delay spread on the choice of subarray, we have considered a channel with a relatively smaller delay spread. In Fig. 5, we refer to the eight-path channel with delay spread $\left[\begin{array}{ll}0 & 3.2 \mu \mathrm{s}\end{array}\right]$ (exponential pdp) as channel (a) and the eight-path channel with delay spread $\left[\begin{array}{ll}0 & 7.4 \mu \mathrm{s}\end{array}\right]$ (exponential pdp) as channel (b). Since the eigenvalue spread of the sampled Fourier transform matrix reduces with the increase in channel delay spread, the $\operatorname{MSE}(\tau)$ for the large delay spread channel [channel (b)] reduces at a faster rate compared with that for a smaller delay spread [channel (a)]. The increase in $\operatorname{MSE}(\tau)$ for large subarray lengths is due to the lack of autocorrelation averaging. Note that this is more evident in the simulation, since the channel taps are correlated in time. This plot helps us in fixing the subarray length given the channel delay spread. In further simulations, we fix the subarray length to be $K_{s}=30$, and the number of OFDM symbols for autocorrelation averaging is taken to be $N=50$.

Fig. 6 compares the MSE performance of the proposed subspace (SS) method and the PR mLS (PRmLS) pilot databased channel-estimation methods with CCI with pilot SIR $\kappa_{p}=0$ and $6 \mathrm{~dB}$. The MSE is evaluated after averaging the autocorrelation over $n=50$ OFDM symbols. The performance of the PRmLS-based method is evaluated based on the channel interpolation method, as explained in [25]. Note that the channel estimates derived from the preamble symbol become obsolete with time due to Doppler. Hence, for the OFDM symbols that are away from the preamble symbol (in the frame), the channel is only estimated from 53 pilots. Therefore, it is observed that PRmLS suffers from an irreducible error floor. The loss in performance of the PRmLS method is due to the following:

1) interference from other $\mathrm{BS}$;

2) interpolation errors due to the nonsampled nature of the channel, which are introduced by the DFT-based interpolators.

The plot shows a significant improvement in performance of the subspace-based channel estimation technique over the PRmLS-based method. The subspace-based method estimates the multipath delay locations of the desired and interferer channels and projects the pilot channel estimates onto the basis derived from the multipath delay information. Since the multipath delay locations of the desired and interferer channels are assumed to be different, high-quality channel estimation is possible. The delays are tagged using only one preamble symbol transmitted at the start of the frame. We have also plotted the MSE for the receiver with ideal multipath delays and ideal delay tagging. It is clear that the MSE of the proposed method follows the MSE with ideal delays for the operating SNR region. The effect of interference on MSE dominates over noise at higher SNR regions and, hence, the MSE of the subspace-based method floors. The effect of interference on MSE is a function of the minimum separation between the multipath delays and is discussed in detail in Fig. 8.

Fig. 7 compares the performance of channel estimation techniques for a coded OFDM system. The pilot and data SIRs are set at $\kappa_{p}=0, \kappa_{d}=0,6 \mathrm{~dB}$. It is clear from the plot that the MMSE-DC detection scheme using the channel estimates obtained from the proposed SS-based method outperforms the other schemes. Since INC is the zero-forcing solution, the performance remains unchanged with the SIR variation on the data carriers. The PRmLS-based detection method completely fails due to its inability to estimate the channel with severe interference.

Figs. 8 and 9 provide the MSE and the coded bit error probability performance by varying the minimum spacing between the paths of the desired and interferer multipath delays $(\delta \tau)$ for $\kappa_{d}=0 \mathrm{~dB}$. The multipath channels were generated using an exponential power-delay profile. The multipath delays of the desired and interferer channels were randomly generated over the interval $\left(\left[\begin{array}{ll}0 & 7.4 \mu \mathrm{s}\end{array}\right]\right)$ such that their minimum delay spacing is maintained. An MMSE-DC-based receiver is used for the simulations. The MSE of the channel estimates is a function of noise and interference, and the closer the multipath delays of the desired and interferer channels, the more the effect of interference on the channel estimates. This is due to the nonorthogonality of the delay subspaces spanned by the desired and interferer channels. ${ }^{6}$ However, observe that when the minimum spacing between the multipath delays $\delta \tau$ is made large, the delay subspaces become "nearly" orthogonal, and the error due to interference decreases. Note that even with a minimum spacing of $\delta \tau=0$, we are able to achieve a better performance compared with the PRmLS method.

\section{CONCLUSION}

We have presented a novel subspace-based channel estimation and tracking method in the presence of strong CCI for cellular reuse-1 OFDM systems such as the IEEE $802.16 \mathrm{~d} / \mathrm{e}$ WMAN [1]. In such reuse-1 systems, the pilot subcarrier positions of the desired and interferer BSs overlap, which severely corrupts the channel estimates, particularly for users at the sector or cell edge. While the use of PR pilot sequences for interference rejection is proposed in [1], this results in an

\footnotetext{
${ }^{6}$ For sample-spaced channel models with distinct multipath delays, the delay subspace spanned by the desired and interferer channels are orthogonal.
} 
irreducible channel-estimation error, resulting in severe degradation in the BER performance when the interference is strong. The proposed channel estimation method can estimate and track both desired and interfering channels with a significantly lower MSE, provided that the multipath delay locations of the desired and interferer channels do not exactly overlap. We assume that the time-of-flight difference between the desired and interfering signals ensures that the multipath locations of the corresponding channels are distinct. Moreover, simulation results are provided with randomly generated multipath delays with their minimum separation equal to zero to show the efficacy of the proposed method. We have analytically derived the MSE expression for the estimated multipath delays and found that the simulation and analysis closely matches. Given the approximate pdp, the MSE analysis of the multipath delays can help in reducing the receiver complexity by minimizing the subarray length for satisfactory system performance. The simulation results provided indicate that accurate channel estimates can greatly improve the error rate performance when interference-suppressing detectors are employed.

\section{REFERENCES}

[1] Standard for Local and Metropolitan Area Networks Part 16: Air Interface for Fixed Broadband Wireless Access Systems, IEEE P802.16-2004, 2004.

[2] Draft Table of Content for the IEEE $802.16 \mathrm{~m}$ System Description Document, Mar. 2008. IEEE C80216m-08/702r3.

[3] Y. Zhang and H.-H. Chen, Mobile WiMAX: Toward Broadband Wireless Metropolitan Area Networks. Boca Raton, FL: CRC, 2007.

[4] A. Ferneke, A. Klein, B. Wegmann, K. Dietrich, and E. Humburg, "Performance of IEEE 802.16e OFDMA in tight reuse scenarios," in Proc. IEEE PIMRC, Sep. 2007, pp. 1-5.

[5] Y. Li and N. R. Solenberger, "Adaptive antenna arrays for OFDM systems with co-channel interference," IEEE Trans. Commun., vol. 47, no. 2, pp. 217-229, Feb. 1999.

[6] G. Luca, A. Luici, P. Giovanni, O. Shuktai, M. Okada, and K. Shozo, "Co-channel interference cancellation based on MIMO-OFDM systems," Wireless Commun., vol. 9, no. 6, pp. 8-17, Dec. 2002.

[7] A. Jeremic, T. A. Thomas, and A. Nehorai, "OFDM channels estimation in the presence of interference," IEEE Trans. Signal Process., vol. 52, no. 12, pp. 3429-3439, Dec. 2004.

[8] M. Munster, T. Keller, and L. Hanzo, "Co-channel interference suppression assisted adaptive OFDM in interference limited environments," in Proc. IEEE VTC-Fall, Sep. 1999, vol. 1, pp. 284-288.

[9] J. Li, K. B. Letaief, and Z. Cao, "Co-channel interference cancellation for space-time coded OFDM systems," IEEE Trans. Wireless Commun., vol. 2, no. 1, pp. 41-49, Jan. 2003.

[10] S. Dieter, G. Matz, H. Franz, and P. Loubaton, "MMSE estimation of time varying channels for DVB-T systems with strong co-channel interference," in Proc. EUSIPCO, Sep. 2002, vol. 3, pp. 25-28.

[11] T. Hunziker, M. Hashiguchi, and T. Ohira, "Reception of coded OFDM signals in broad-band fading environments with strong cochannel interference," in Proc. IEEE GLOBECOM, Dec. 2003, vol. 4, pp. 2310-2314.

[12] C.-S. Ni and K.-C. Chen, "Cochannel interference suppression for coded OFDM systems over frequency-selective slowly fading channels," in Proc. IEEE VTC-Fall, Sep. 2004, vol. 1, pp. 679-683.

[13] J. J. van de Beek, O. Edfors, M. Sandell, S. K. Wilson, and P. O. Borjesson, "On channel estimation in OFDM systems," in Proc. IEEE VTC, Jul. 1995, vol. 2, pp. 815-819.

[14] B. Yang, K. B. Letaief, R. S. Cheng, and Z. Cao, "Channel estimation for OFDM transmission in multipath fading channels based on parametric channel modeling," IEEE Trans. Commun., vol. 49, no. 3, pp. 467-479, Mar. 2001.

[15] Y. Li and L. J. Cimini, "Bounds on the interchannel interference of OFDM in time-varying impairments," IEEE Trans. Commun., vol. 49, no. 3, pp. 401-404, Mar. 2001.

[16] M. R. Raghavendra and K. Giridhar, "Improving channel estimation in OFDM systems for sparse multipath channels," IEEE Signal Process. Lett., vol. 12, no. 1, pp. 52-55, Jan. 2005.
[17] O. Simeone, Y. Bar-Ness, and U. Spagnolini, "Pilot-based channel estimation for OFDM systems by tracking the delay-subspace," IEEE Trans. Wireless Commun., vol. 3, no. 1, pp. 315-325, Jan. 2004.

[18] M. R. Raghavendra, E. Lior, S. Bhashyam, and K. Giridhar, "Parametric channel estimation for pseudo-random subcarrier-allocation in uplink OFDMA," IEEE Trans. Signal Process., vol. 55, no. 11, pp. 5370-5381, Nov. 2007.

[19] R. Negi and J. Cioffi, "Pilot tone selection for channel estimation in a mobile OFDM system," IEEE Trans. Consum. Electron., vol. 44, no. 3 , pp. 1122-1128, Aug. 1998.

[20] S. Ohno and G. B. Giannakis, "Capacity maximizing MMSE-optimal pilots for wireless OFDM over frequency-selective block Rayleigh-fading channels," IEEE Trans. Inf. Theory, vol. 50, no. 9, pp. 2138-2145, Sep. 2004.

[21] R. Roy and T. Kailath, "ESPRIT-Estimation of signal parameters via rotational invariance techniques," IEEE Trans. Acoust., Speech, Signal Process., vol. 37, no. 7, pp. 984-995, Jul. 1989.

[22] S. U. Pillai and B. H. Kwon, "Forward/backward spatial smoothing techniques for coherent signal identification," IEEE Trans. Acoust., Speech, Signal Process., vol. 37, no. 1, pp. 8-15, Jan. 1989.

[23] T. J. Shan, M. Wax, and T. Kailath, "On spatial smoothing for directionof-arrival estimation of coherent signals," IEEE Trans. Acoust., Speech, Signal Process., vol. ASSP-33, no. 4, pp. 806-811, Aug. 1985.

[24] H. L. Van Trees, Optimum Array Processing, Part 4 of Detection, Estimation, and Modulation Theory. Hoboken, NJ: Wiley, 2002.

[25] Y. Li, L. J. Cimini, Jr., and N. R. Sollenberger, "Robust channel estimation for OFDM systems with rapid dispersive fading channels," IEEE Trans. Commun., vol. 46, no. 7, pp. 902-915, Jul. 1998.

[26] M. Z. Win and J. H. Winters, "Analysis of hybrid selection/maximal-ratio combining in Rayleigh fading," in Proc. IEEE ICC, 1999, vol. 1, pp. 6-10.

[27] B. Xia and J. Wang, "Effect of channel-estimation error on QAM systems with antenna diversity," IEEE Trans. Commun., vol. 52, no. 12, p. 2209, Dec. 2004.

[28] W. C. Jakes, Microwave Mobile Communications. Piscataway, NJ: IEEE Press, 1993.

[29] U. Reimers, "Digital video broadcasting," IEEE Commun. Mag., vol. 36, no. 6, pp. 104-110, Jun. 1998.

[30] J. G. Proakis, Digital Communications, 3rd ed. New York: McGrawHill, 1995.

[31] L. Hanzo, M. Munster, B. J. Choi, and T. Keller, OFDM and MC-CDMA for Broadband Multi-User Communications, WLANs and Broadcasting. Piscataway, NJ: IEEE Press, 2003.

[32] B. D. Rao and K. V. S. Hari, "Performance analysis of ESPRIT and TAM in determining the direction of arrival of plane waves in noise," IEEE Trans. Acoust., Speech, Signal Process., vol. 37, no. 12, pp. 1990-1995, Dec. 1989.

[33] B. D. Rao and K. V. S. Hari, "Effect of spatial smoothing on state space methods/esprit," in Proc. 5th ASSP Workshop Spectrum Estimation Model., 1990, pp. 377-381.

[34] P. Stoica and A. Nehorai, "Performance comparison of subspace rotation and music methods for direction estimation," IEEE Trans. Signal Process., vol. 39, no. 2, pp. 446-453, Feb. 1991

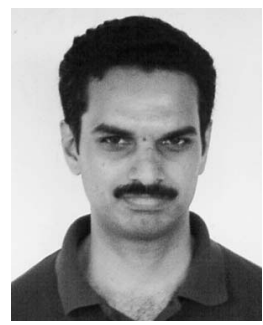

M. R. Raghavendra received the B.E. degree in telecommunications engineering from Bangalore University, Bangalore, India, in 2001 and the Ph.D. degree from the Indian Institute of Technology Madras (IIT Madras), Chennai, India, in 2007.

$\mathrm{He}$ was a Research Scientist with the Centre for Wireless Communications, University of Oulu, Oulu, Finland, from October 2007 to September 2008. Since October 2008, he has been a Senior Engineer with Motorola India Electronics Private Ltd., Bangalore, where he works on the physical layer aspects of 3GPP-LTE. His research interests include wireless communications and signal processing for communications. 


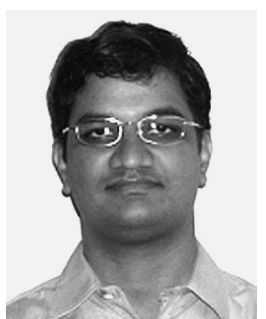

Srikrishna Bhashyam received the B.Tech. degree in electronics and communications engineering from the Indian Institute of Technology Madras (IIT Madras), Chennai, India, in 1996 and the M.S. and $\mathrm{Ph} . \mathrm{D}$. degrees in electrical and computer engineering from Rice University, Houston, TX, in 1998 and 2001, respectively.

Between June 2001 and March 2003, he was a Senior Engineer with Qualcomm CDMA Technologies, Campbell, CA. Since May 2003, he has been an Assistant Professor with the Department of Electrical Engineering, IIT Madras. His current research interests are in resource allocation, adaptive transmission, code design, and information theory for multiterminal wireless communication systems.

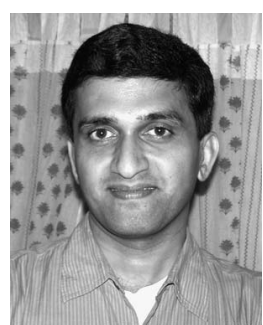

K. Giridhar received the B.Sc. degree in applied sciences from the PSG College of Technology, Coimbatore, India, in 1985, the M.E. degree in electrical communications engineering from the Indian Institute of Science, Bangalore, India, in 1989, and the $\mathrm{Ph} . \mathrm{D}$. degree in electrical and computer engineering from the University of California, Santa Barbara, in 1993.

He was a Visiting Faculty with Sri Sathya Sai University, Prasanthi Nilayam, India. After working for a year as a Research Affiliate with Stanford University, Stanford, CA, he joined the Indian Institute of Technology Madras (IIT Madras), Chennai, India, in 1994, where he is currently a Professor of electrical engineering. Being an active member of the TeNeT group, his research interests are in the areas of adaptive estimation with a focus on multipleinput-multiple-output orthogonal frequency division multiplexing wireless transceivers and in the performance analysis of wireless networks. His research group also works closely with the Center of Excellence in Wireless Technology. 